\title{
At the helm of the NIDDK
}

A of April 2007, Griffin P. Rodgers has taken over as director of the NIDDK, but had been the deputy director under Allen Spiegel since 2001 and the chief of Hematology since 1998. The JCI took the opportunity to ask this career investigator in the $\mathrm{NIH}$ about his goals and visions for the NIDDK.

JCI: You moved quickly from chief of Hematology to deputy director of the NIDDK to director - what do you think contributed to this ascent?

Rodgers: NIH is an incredibly supportive environment. I had terrific encouragement and great mentorship throughout my career and I owe a great deal to the people who have guided me along the way. As time passed, I became very interested in the challenges of managing a large research organization, and I pursued opportunities to augment my laboratory experiences along those lines.

JCI: What areas of research do you see expanding while you serve as director? Are there areas in which you want to invite grants and proposals?

Rodgers: We are extremely interested in translational research. Other areas of interest would focus on interactions between genetic variations and the environment, encompassing different types of environmental triggers of, or contributors to, disease, as well as research related to an individual's response to potential disease treatments. Additional examples of cross-cutting research interest are metabolomics, proteomics, and research on the gut microbiome.

JCI: Given the increasing attention on obesity, do you have any comment on what the NIDDK will do to combat the "epidemic"? Do you plan any increased funding or outreach programs or education incentives?

Rodgers: It is clearly a critical - and very complex - public health problem. Research areas that we fund include basic, clinical, and translational research toward prevention and treatment of obesity, as well as studies addressing their link to many other serious diseases and disorders within our institute's mission, such as type 2 diabetes, nonalcoholic steatohepatitis, and urinary incontinence.

The NIDDK's support of basic, clinical, and translational research, as well of as education and outreach efforts, will be important contributors to multidimensional, national efforts to combat obesity.
We support scientists who are elucidating the molecular regulation of appetite, energy expenditure, and energy storage, because this type of basic research opens new avenues for treatment approaches that could be pursued by academic and other researchers. We also support scientists who are studying behavioral and environmental factors that contribute to excess weight gain.

With respect to education and outreach programs, one example is a multicultural program we developed to disseminate the results of the lifestyle intervention of our Diabetes Prevention Program clinical trial. This trial demonstrated that moderate weight loss, from a more healthful diet and moderate exercise, can dramatically

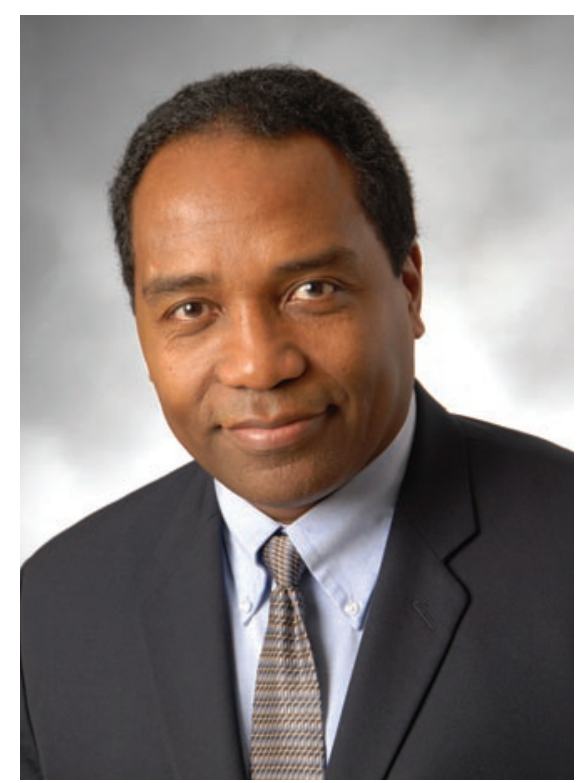

Griffin Rodgers, new director of the NIDDK

reduce risk for type 2 diabetes in those at high risk.

JCI: Then given your particular experience as a minority, are you sensitive to increased efforts in support of programs geared toward minority applicants or toward the study of minority groups?

Rodgers: Many of the diseases within the NIDDK mission disproportionately affect ethnic minority populations. From my own experience, including my research on sickle cell disease, I am keenly aware of the importance and benefits of the participation of minorities in research - as investigators and as patients in clinical studies. I plan to build upon the institute's already strong commitment to involving minorities in our research efforts to combat conditions that place a disproportionate burden on these groups. The institute has been dedicated to ensuring that its clinical studies include substantial minority participation. Minorities have been involved as investigators, as well as study participants, in working to highlight and address this health disparity. In recent years, the institute helped to support the largest clinical trial to date in African Americans with kidney disease, identifying a therapy to slow disease caused by hypertension in this group.

JCI: With a flat NIH budget for the next year, and ever-increasing costs and numbers of applications, what are your proposed solutions to keep research afloat and young scientists engaged?

Rodgers: With careful planning and anal$y$ sis, and with continued valuable external advice from investigators, health care professionals, patient advocates, and others, we will strive to deploy our budgetary resources in the most effective ways to advance research progress. Several overarching principles will guide my leadership as our institute moves forward. These include: maintaining a vigorous investigator-initiated research portfolio; supporting pivotal clinical studies and clinical trials; preserving a stable pool of talented new investigators; fostering exceptional research training and mentoring opportunities; and ensuring knowledge dissemination through outreach and communications.

To expand upon the principles relating to new investigators, I believe that these scientists are critical for carrying out biomedical research in the future. Moreover, their new ideas and fresh perspectives can also invigorate the research enterprise now. Thus, we want to ensure that today's new scientists view research as a viable career and that they can realize their potential for advancing research progress. The NIDDK participates in NIH-wide efforts to bolster support for new investigators, and our institute also promotes additional mentoring opportunities and special funding consideration for talented new investigators.

\section{Ushma S. Neill}

